\title{
SRT1720 promotes survival of aged human mesenchymal stem cells via FAIM: a pharmacological strategy to improve stem cell-based therapy for rat myocardial infarction
}

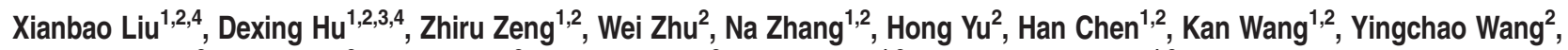 \\ Lengmei Wang ${ }^{2}$, Jing $\mathrm{ZhaO}^{2}$, Ling Zhang ${ }^{2}$, Rongrong $\mathrm{Wu}^{2}$, Xinyang $\mathrm{Hu}^{1,2}$ and Jian'an Wang, ${ }^{*, 1,2}$
}

\begin{abstract}
SIRT1 has been proved to rejuvenate and improve the therapeutic efficacy of aged rat mesenchymal stem cells (MSCs). Herein, we investigate the protective effect of pretreatment with SIRT1 activator SRT1720 on aged human MSCs (hMSCs). The optimized pretreatment condition for aged hMSCs was determined to be $0.5 \mu \mathrm{M}$ SRT1720 for $24 \mathrm{~h}$ by monitoring the survival of aged hMSCs subjected to serum deprivation \pm hypoxia and $\pm 500 \mu \mathrm{M}$ hydrogen peroxide $\left(\mathrm{H}_{2} \mathrm{O}_{2}\right)$. Pretreatment with these conditions increased the survival of aged hMSCs 1 day (2.7-fold) and 3 days (1.9-fold) after being transplanted into a rat myocardial infarction (MI) model created by ligation of the left anterior descending (LAD) coronary artery. Transplantation with SRT1720 pretreated aged hMSCs achieved increased left ventricular ejection fraction $(58.9 \pm 3.6$ versus $52.8 \pm 5 \%)$ and angiogenesis with reduced fibrosis of rat hearts as compared to DMSO pretreated group 28 days following MI. Unbiased transcriptome analysis conducted on aged hMSCs under oxidative stress indicated the Fas apoptosis inhibitory molecule (FAIM) was significantly upregulated following SRT1720 pretreatment (14.9 \pm 0.2 -folds). Moreover, the anti-apoptotic effect of SRT1720 was mitigated by FAIM knockdown with a small interfering RNA-targeted FAIM. These results indicated that pretreatment with SRT1720 improves survival of aged hMSCs, and enhances their therapeutic efficacy for rat myocardial infarction (MI). Upregulation of FAIM possibly involves in the mechanisms of the protective effects.
\end{abstract}

Cell Death and Disease (2017) 8, e2731; doi:10.1038/cddis.2017.107; published online 6 April 2017

Transplantation of MSCs has been shown to be safe and is a promising strategy for the treatment of heart diseases. ${ }^{1-4}$ However, survival and engraftment of MSCs following transplantation remains low, thus representing a major barrier for the overall therapeutic efficacy and utility of this approach. Moreover, it has been reported that MSCs from aged donors display a reduced ability to repair the heart in animal models. ${ }^{5,6}$ The biological properties of human MSCs (hMSCs) including proliferation, differentiation potential and stress resistance decline with age and may limit the applications of these cells for clinical therapy. ${ }^{7-9}$ Thus, it's a big challenge to overcome the age-related dysfunction of hMSCs.

Silent information regulator 2 homolog 1 (SIRT1), also known as sirtuin 1 , is a $\mathrm{NAD}^{+}$dependent histone deacetylase that has important roles in metabolism and age-related pathologies including type 2 diabetes and neurodegenerative diseases. ${ }^{10,11}$ SIRT1 has also been shown to positively regulate cell survival and apoptosis, as well as the responses to stress and inflammation through non-histone targets such as p53, FOXOs and NF-kB. ${ }^{12-14}$ In our previous study, we demonstrated that overexpression of SIRT1 conferred rejuvenation of aged rat MSCs and supported improved therapy in a rat MI model. ${ }^{15,16}$ The findings support a practical strategy to rejuvenate and improve cell therapy by aged hMSCs via augmentation of SIRT1.

As the natural compound resveratrol was identified as a SIRT1 activator, a large number of small molecules have been found to activate SIRT1 (refs 17,18 of which SRT1720 is the most effective and specific one. ${ }^{19}$ SRT1720 treatment extends the lifespan of both healthy mice and those on high fat diets, ${ }^{20,21}$ and can ameliorate the disturbed flow induced senescence of endothelial cells. ${ }^{22}$ However, little is known on the effects of SRT1720 on normal human cells including human MSCs.

In the present study, we determined that SIRT1 expression is downregulated in aged hMSCs and this correlates with an impaired ability to resist stress. We further demonstrated that pretreatment of aged hMSCs with SRT1720 conferred improved cell survival and enhanced therapy in a rat $\mathrm{MI}$ model. Our results support a role for the Fas apoptosis inhibitory molecule (FAIM), in mediating the positive survival and protherapeutic actions of SRT1720 pretreatment by aged hMSCs.

\section{Results}

Characterization of aged hMSCs. Cell surface markers of the hMSCs were determined by flow cytometry. Nearly all of

\footnotetext{
${ }^{1}$ Department of Cardiology, Second Affiliated Hospital, Zhejiang University School of Medicine, Hangzhou, Zhejiang, China; ${ }^{2}$ Provincial Key Laboratory of Cardiovascular Research, Hangzhou, Zhejiang, China and ${ }^{3}$ Department of Cardiology, Ningbo Medical Center Lihuili Eastern Hospital, Ningbo, China

*Corresponding author: J Wang, Department of Cardiology, Second Affiliated Hospital, Zhejiang University School of Medicine, 88 Jiefang Road, Hangzhou, Zhejiang 310009, China. Tel: +86 5718731 5001; Fax: +86 5718703 7885; E-mail: jian_an_wang@yahoo.com

${ }^{4}$ These authors contributed equally to this work.

Received 04.10.16; revised 24.1.17; accepted 03.2.17; Edited by D Aberdam
} 
a

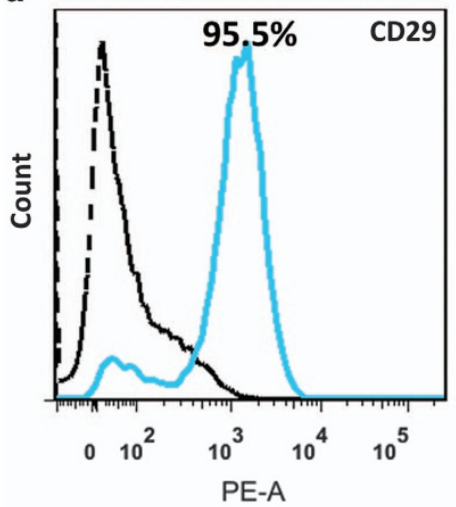

d

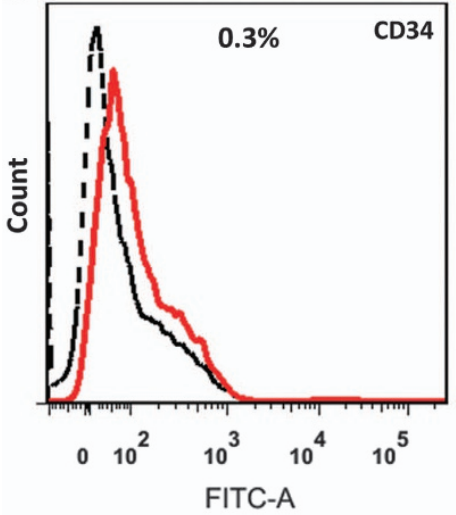

f

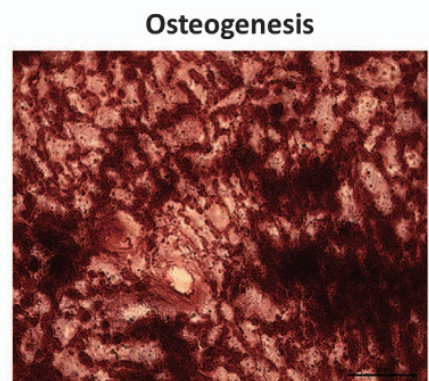

b

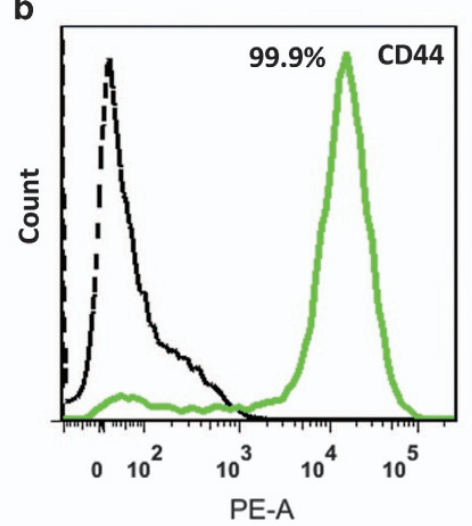

e

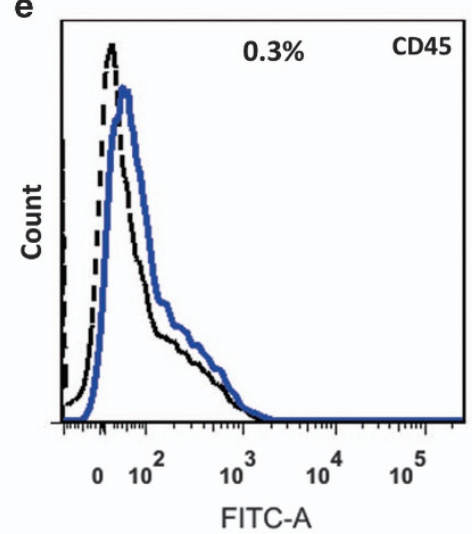

g

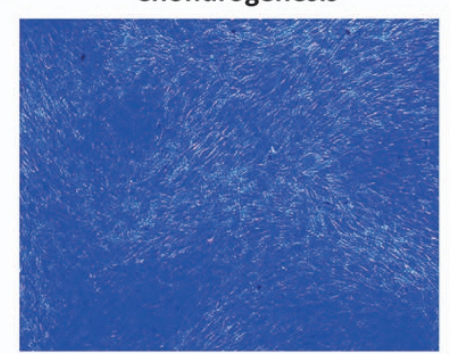

c

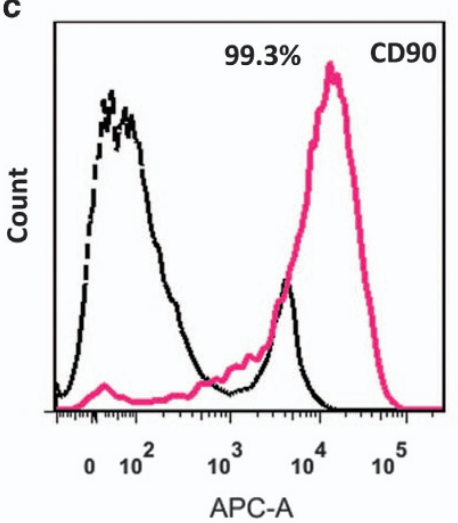

h

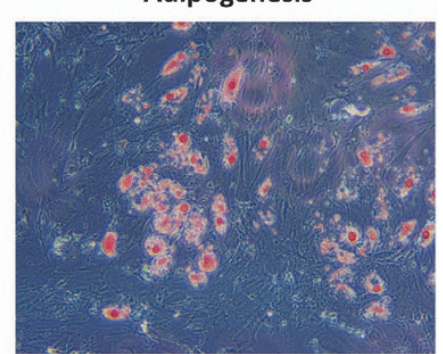

Figure 1 Characterization of hMSCs. Nearly all of the cells acquired expressed the cell surface markers PE-CD29 (a), PE-CD44 (b), APC-CD90 (c), and negative for the endothelial cell surface marker FITC-CD34 (d) and hematopoietic surface marker FITC-CD45 (e). The osteogenesis, chondrogenesis and adipogenesis differentiation of hMSCs was induced and visualized by alizarin red staining (f, dark red), toluidine blue staining (g, dark blue), and oil red $\mathrm{O}$ staining (h, red), respectively

the cells acquired in our study were positive for the mesenchymal stem cell (MSC) surface markers: CD29, CD44, CD90 and negative for the endothelial cell surface marker CD34 and hematopoietic surface marker CD45, indicating characteristics of MSCs (Figures 1a-e). In addition, the multi-lineage differentiation capacity of hMSCs has also been tested, and they were proved to be able to differentiate into osteocytes, chondrocytes, and adipocytes (Figures 1f-h).

Comparison of young and aged hMSCs reveals an association of SIRT1 with declined performances of aged hMSCs. In our previous studies, we have demonstrated that aged rat MSCs performed more expression of the cell senescence marker, $\beta$-galactosidase and weaker resistance to peroxide stress coupling with the low expression and activity of SIRT1 compared with young rat MSCs. ${ }^{15,16}$ Similarly, expression of $\beta$-galactosidase was increased in aged hMSCs compared with young cells (OMSC 10.2 \pm 4.3 versus YMSC $2.7 \pm 1 \%, P=0.03$ ) (Supplementary Figures $1 \mathrm{a}$ and b). To investigate a possible relationship between SIRT1 and cell viability of young versus aged hMSCs, we evaluated cell survival under conditions of imposed oxidative stress induced by serum deprivation combined with $500 \mu \mathrm{M}$ hydrogen peroxide $\left(\mathrm{H}_{2} \mathrm{O}_{2}\right)$. Resistance to stress in aged hMSCs was significantly decreased compared to young hMSCs as indicated by survival indices (OMSC $44.6 \pm 4.7$ versus YMSC $61.2 \pm 3.6 \%, P=0.03$ ) (Supplementary Figures 1c). Aged hMSCs also displayed decreased expression and activity of SIRT1 (Supplementary Figures $1 \mathrm{~d}$ and e). These results 
indicate a correlation between SIRT1 expression and resistance to stress in aged hMSCs.

SRT1720 improves the survival of aged hMSCs in vitro and in vivo. To investigate whether decreased activity of SIRT1 was responsible for the reduced stress resistance of aged hMSCs, we used a specific activator of SIRT1, SRT1720, to stimulate SIRT1 activity in these cells. To mimic the low nutrition and hypoxic environment in the ischemic heart, three in vitro stress models were established and used to evaluate cell survival. The models include: serum deprivation, serum deprivation under hypoxia, and serum deprivation combined with $\mathrm{H}_{2} \mathrm{O}_{2}$. Respectively, cells were exposed to $24 \mathrm{~h}$ of serum deprivation or serum deprivation plus hypoxia, a time where $\sim 50 \%$ cells survived the latter condition compared to normal culture without stress (Supplementary Figures $2 \mathrm{a}$ and $\mathrm{b}$ ). In the setting of serum deprivation combined with $\mathrm{H}_{2} \mathrm{O}_{2}$, a concentration of $500 \mu \mathrm{M} \mathrm{H}_{2} \mathrm{O}_{2}$ for $3 \mathrm{~h}$ was selected to induce $\sim 50 \%$ cell survival compared to normal culture without stress (Supplementary Figure 2c). In these models, under the serum deprivation condition, SRT1720 pretreatment exhibited a dose-dependent protective effect on aged hMSCs, with the highest survival rate reached at a concentration of $0.2 \mu \mathrm{M}$ SRT1720 $(73.0 \pm 2.5 \%)$ with no further benefits observed at higher concentrations (Figure 2a). However, when aged hMSCs were exposed to serum free medium combined with either hypoxia or $500 \mu \mathrm{M}$ $\mathrm{H}_{2} \mathrm{O}_{2}$, a concentration of $0.5 \mu \mathrm{M}$ was needed for maximal survival benefit $(72.4 \pm 7.5 \%$ under hypoxia, $67.0 \pm 4.2 \%$ under $\mathrm{H}_{2} \mathrm{O}_{2}$ stress) (Figures $2 \mathrm{~b}$ and $\mathrm{c}$ ). Therefore, a concentration of $0.5 \mu \mathrm{M}$ was determined to be the optimized concentration of SRT1720 treatment for aged hMSCs. We further found that $24 \mathrm{~h}$ of pretreatment with $0.5 \mu \mathrm{M}$ SRT1720 was required to achieve maximum survival of aged hMSCs cultured in serum deprivation combined with $500 \mu \mathrm{M} \mathrm{H}_{2} \mathrm{O}_{2}$ $(88 \pm 9.6 \%$; Figure $2 d)$.

To determine whether enhanced survival in vitro translated into improved survival in vivo, SRT1720 or vehicle pretreated aged hMSCs were injected into infarcted rat hearts and the male sex determination gene sry copy number within the recipient hearts was measured at 1, 3 and 28 days, respectively, post-transplantation. No sry was detected in samples from sham and DMEM groups. However, relative to controls (DMSO pretreated group), a 2.7- and 1.9-fold increase in the copy number of sry was observed in the SRT1720 pretreated group at 1 and 3 days, respectively (Figures $2 e$ and f). The sry was not detected in any samples at 28-day post-transplantation. These results indicate that pretreating aged hMSCs with SRT1720 promotes cell survival at early times after transplantation in the ischemic rat heart.

Enhanced angiogenesis by SRT1720 pretreatment. Rat hearts were collected at 28 days after $\mathrm{MI}$ and cell therapy, and angiogenesis was assessed by quantifying blood vessel density around the infract zone using immunofluorescence staining of CD31 (a marker for vascular endothelial cells) and a-SMA (a marker for vascular smooth muscle cells) (Figures $3 a$ and $c$ ). The density of CD31 and $a$-SMA staining were slightly increased in the DMSO-OMSC group and significantly increased in the SRT1720-OMSCs group (Figures $3 b$ and $d$ ). These results indicate that pretreatment with SRT1720 enhanced the pro-angiogenic properties of aged hMSCs perhaps secondarily to improved survival and early engraftment.
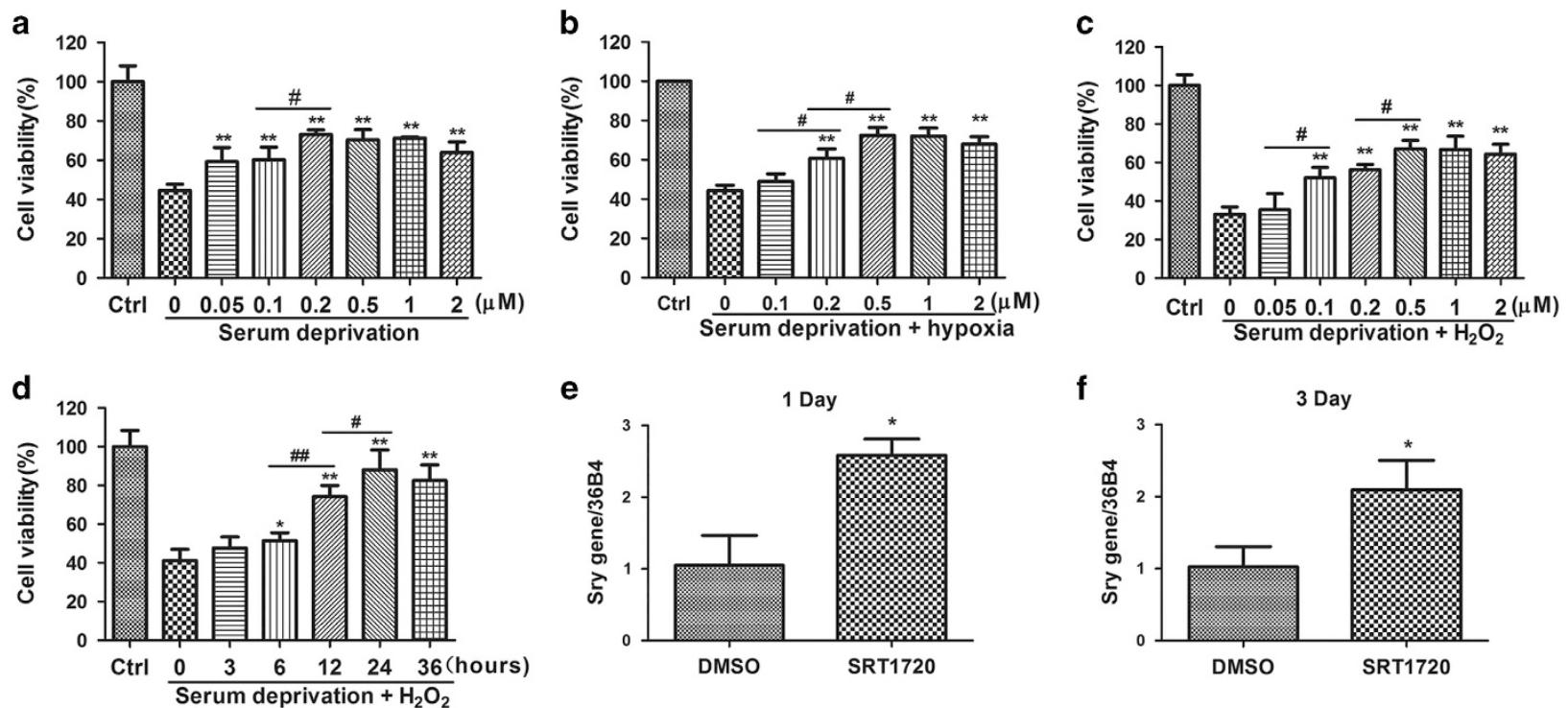

Figure 2 SRT1720 pretreatment promotes the survival of aged hMSCs under stress in vitro and in vivo. Protective effect of various concentration $(0,0.05,0.1,0.2,0.5,1$, $2 \mu \mathrm{M}$ ) of SRT1720 was evaluated under the situations of serum deprivation (a), serum deprivation and hypoxia (b), serum deprivation and $\mathrm{H}_{2} \mathrm{O}_{2}$ (c) by CCK-8 analysis. The survivals of aged hMSCs pretreated with $0.5 \mu \mathrm{M} \mathrm{SRT} 1720$ for different time $(0,3,6,12,24,36 \mathrm{~h})$ are detected under serum deprivation combined $\mathrm{H}_{2} \mathrm{O}_{2}$ situation (d). $S R Y$ gene expression of hMSCs in the whole heart was analyzed at day 1 (e) and day 3 (f) after cell therapy. The control condition (Ctrl) was normal culture medium (DMEM with $10 \%$ FBS). The volume fraction of DMSO was the same in every groups. Data are expressed by mean \pm S.D. (three independent experiments, $N=3$ ). ${ }^{*} P<0.05$ versus $\mathrm{DMSO}$ group; ${ }^{* *} P<0.01$ versus DMSO group; ${ }^{\#} P<0.05$ 
a

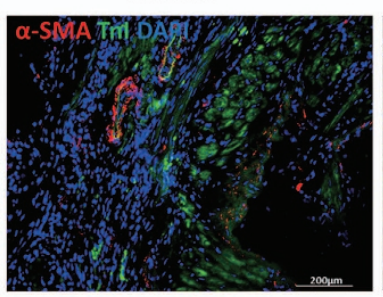

c

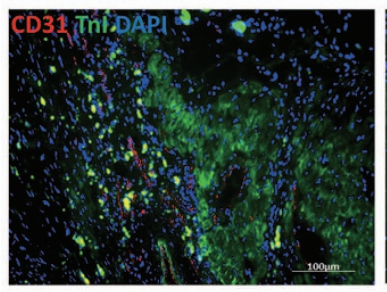

DMSO-OMSC
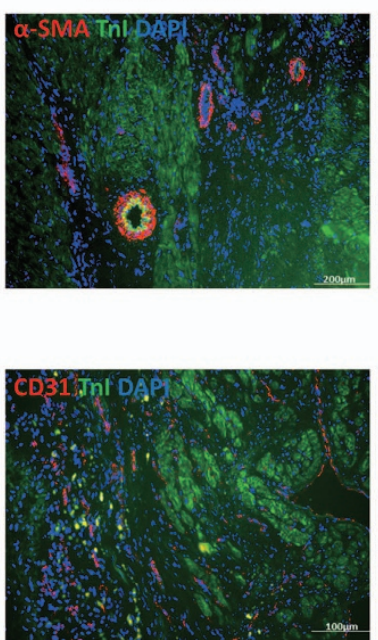

SRT1720-OMSC
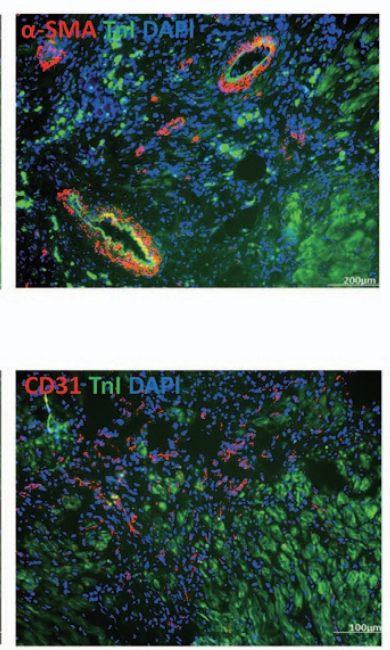

b

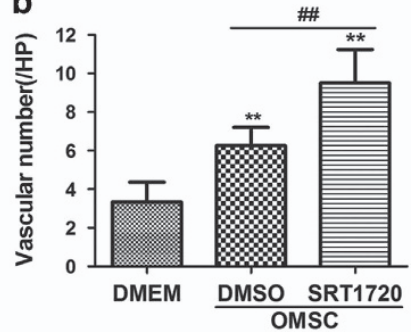

d

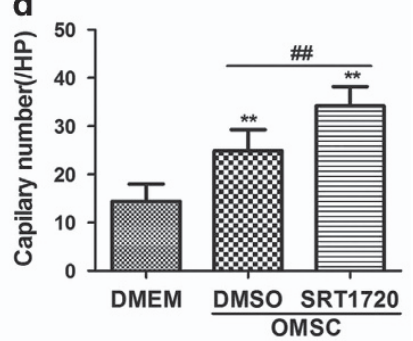

f

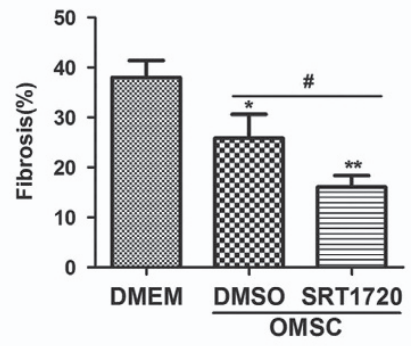

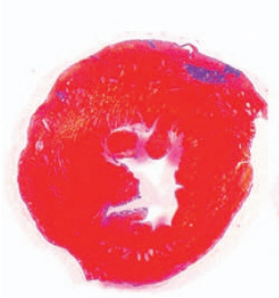

sham

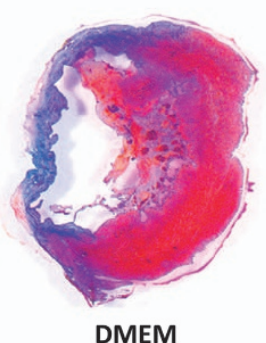

DMEM

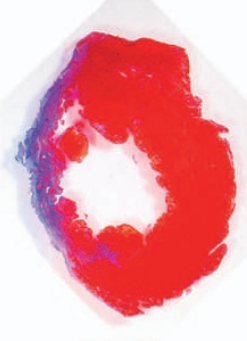

DMSO

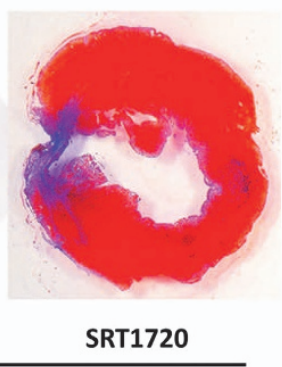

SRT1720

OMSC

Figure 3 Improved angiogenesis and fibrosis of rat hearts after transplantation with SRT1720 pretreated aged hMSCs. Blood vessels in the border zone of ischemic hearts were stained with $\alpha$-SMA (a and $\mathbf{b})$ and CD31 (c and d). Cardiac remodeling was analyzed by Masson's trichrome staining (e and f). Data are expressed by mean \pm S.D. $(N=6)$. ${ }^{*} P<0.05$ versus DMSO group; ${ }^{*} P<0.01$ versus DMSO group; ${ }^{\#} P<0.05 ;{ }^{\# \#} P<0.01$

\begin{abstract}
SRT1720 pretreatment of aged hMSCs confers reduced MI scar size. Myocardial tissue sections at 28 days following cell therapy were stained with Masson's trichrome staining to evaluate the interstitial fibrosis. As expected scar areas were larger in the DMEM treatment group compared to the sham group, but reduced in the hMSCs treated group (Figure 3e). When the collagen volumes were quantified, the scar size were reduced in the DMSO-OMSC group compared to DMEM group (DMSO-OMSC group $25.9 \pm 4.7 \%$ versus DMEM group $38 \pm 3.4 \%, P=0.014$ ) and were reduced with greater significance in the SRT1720-OMSC group (SRT1720-OMSC group $16 \pm 2.3 \%$ versus DMEM group $38 \pm 3.4 \%, P=0.008$ ) (Figure $3 \mathrm{f}$ ). These results suggest that SRT1720 pretreatment of aged hMSCs more effectively alleviates cardiac fibrosis.
\end{abstract}

SRT1720 pretreatment of aged hMSCs leads to improved cardiac function. Transthoracic $\mathrm{M}$ model echocardiography (Figure 4a) was conducted to evaluate cardiac dimensions (left ventricular end diastolic diameter (LVEDD) and left ventricular end systolic diameter (LVESD)) and functions (left ventricular ejection fraction (LVEF) and left ventricular fraction shorting (LVFS)) in the rat hearts following cell therapy.
Compared to sham group, left ventricular dimensions were enlarged following $\mathrm{Ml}$ (LVEDD from $6.43 \pm 0.44 \mathrm{~mm}$ to $8.11 \pm 0.87 \mathrm{~mm}, P<0.001$; LVESD from $3.71 \pm 0.77 \mathrm{~mm}$ to $6.50 \pm 0.96 \mathrm{~mm}, P=0.007)$. Aged hMSC transplantation (DMSO pretreated) resulted in a trend for improvement of the ventricular dimensions compared to the DMEM group (LVEDD from $8.11 \pm 0.87 \mathrm{~mm}$ to $7.57 \pm 1.03 \mathrm{~mm}, P=0.014$; LVESD from $6.50 \pm 0.96 \mathrm{~mm}$ to $5.44 \pm 0.84 \mathrm{~mm}, P=0.1$ ) (Figures $4 \mathrm{~b}$ and c). Notably, cardiac function of rats severely declined following $\mathrm{Ml}$ compared to sham group (LVEF from $71.8 \pm 9.6 \%$ to $38.1 \pm 10.5 \%, \quad P=0.002 ; \quad$ LVFS from $42.7 \pm 9.4 \%$ to $20 \pm 7 \%, P=0.006$ ), and only small improvements were observed following DMSO pretreated aged hMSC transplantation compared with the DMEM group (LVEF from $38.1 \pm 10.5 \%$ to $52.8 \pm 5 \%, P=0.028$; LVFS from $20 \pm 7 \%$ to $27.9 \pm 2.8 \%, P=0.037$; Figures $4 d$ and e). However, when aged hMSCs pretreated with SRT1720 were transplanted into ischemic hearts, the cardiac dimensions were significantly decreased compared to the DMEM group (LVEDD from $8.11 \pm 0.87 \mathrm{~mm}$ to $7.17 \pm 0.64 \mathrm{~mm}, P=0.026$; LVESD from $6.5 \pm 0.96 \mathrm{~mm}$ to $4.92 \pm 0.39 \mathrm{~mm}, P=0.019$; Figures $4 \mathrm{~b}$ and $\mathrm{c}$ ) and, concomitantly, cardiac function significantly improved (LVEF from $38.1 \pm 10.5$ to 


\section{a}
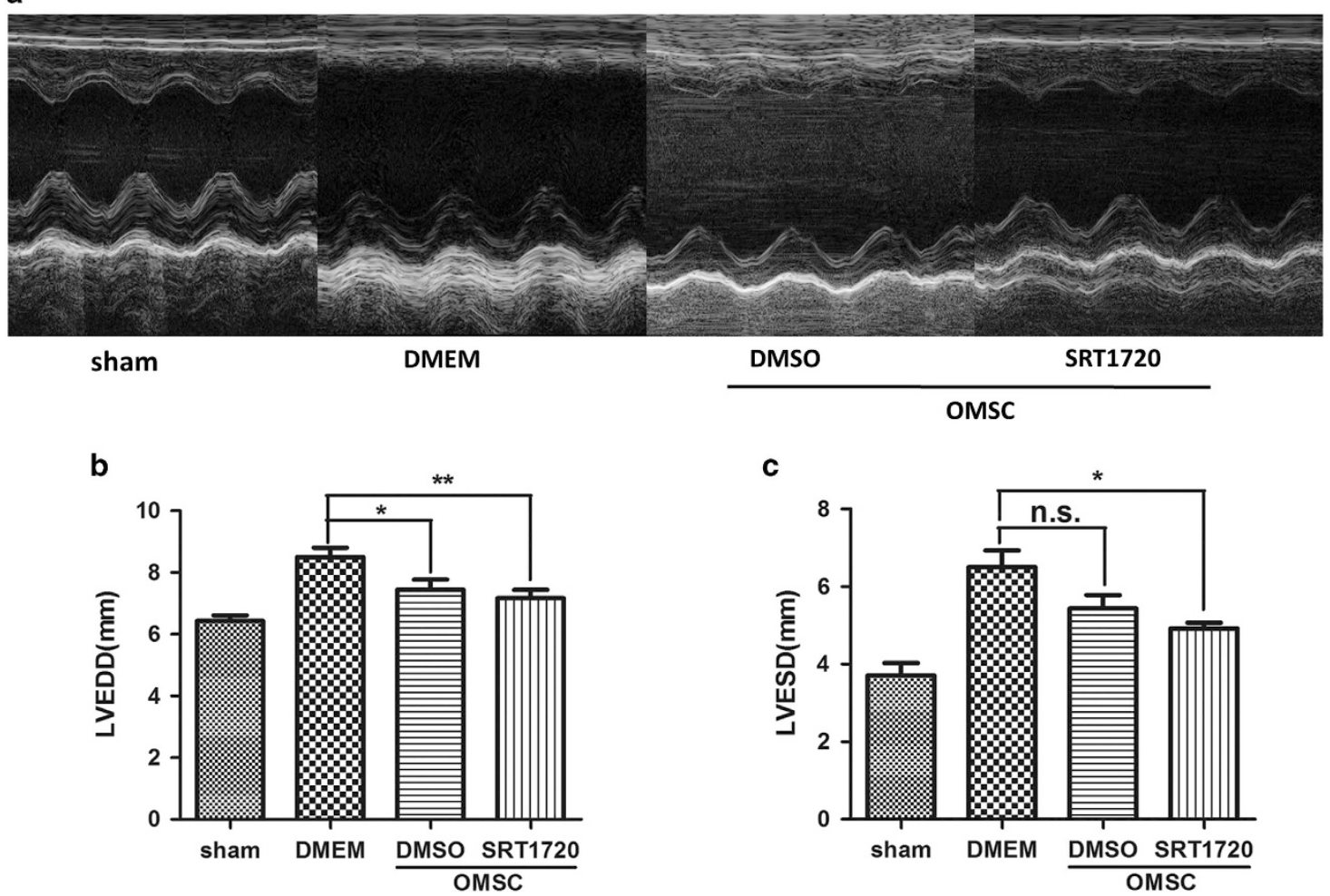

C
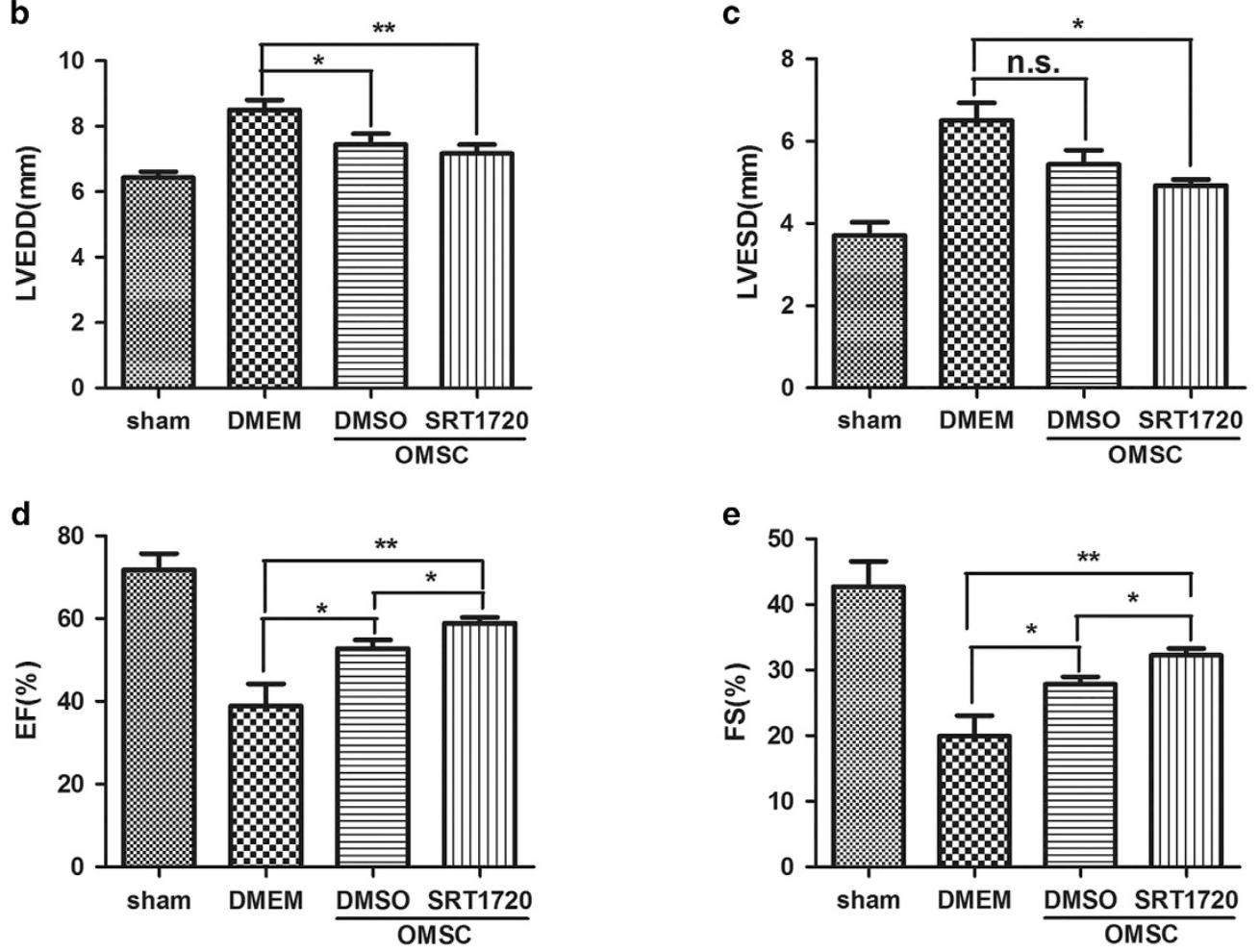

Figure 4 Enhanced cardiac function of rat hearts after SRT1720 pretreated aged hMSCs transplantation. Representative echocardiographic images are shown (a). The $\operatorname{LVEDD~(b),~LVESD~(c),~LVEF~(d)~and~LVFS~(e)~were~analyzed,~respectively.~Data~are~expressed~by~mean~} \pm$ S.D. $(N=6) .{ }^{*} P<0.05 ;{ }^{* \star} P<0.01$

$58.9 \pm 3.6 \%, P=0.009 ;$ LVFS from $20 \pm 7$ to $32.3 \pm 2.7 \%$ $P=0.018$ ) compared to the DMEM group (Figures $4 \mathrm{~d}$ and e).

SRT1720 protects aged hMSCs against apoptosis. To investigate the mechanism leading to improved cell survival following SRT1720 pretreatment of aged hMSCs, we first examined the expression and activity of SIRT1 under the stress condition of serum deprivation combined with $500 \mu \mathrm{M}$ $\mathrm{H}_{2} \mathrm{O}_{2}$. As shown in Figures $5 \mathrm{a}$ and $\mathrm{b}$, no differences of SIRT1 expression were observed under the stresses when aged hMSCs were treated with or without SRT1720 and in the absence or presence of a SIRT1 specific inhibitor, EX-527, compared with DMSO pretreated group. P53 is a SIRT1 substrate that is closely linked to cell survival. ${ }^{13}$ Therefore, we evaluated acetylation of p53, as a surrogate marker of
SIRT1 activity in aged hMSCs. The ratio of acetyl-p53(K382)/ total-p53 was significantly decreased when cells were pretreated with SRT1720 and subsequently increased when pretreated with EX-527 (Figures $5 a$ and c).

To expand upon our understanding of the role of SIRT1 in aged hMSCs, we assessed apoptosis following exposure to serum deprivation plus $500 \mu \mathrm{M} \mathrm{H}_{2} \mathrm{O}_{2}$. The ratio of apoptotic cells detected by Hoechst staining in the SRT1720-OMSCs group was significantly lower than that observed in the DMSOOMSCs group. Moreover, an increased ratio of apoptotic cells was observed upon pretreatment with EX-527 (Figures 5h and i). In keeping with these results, activation of caspase- 3 and caspase-8 were also decreased following SRT1720 pretreatment and conversely enhanced following EX-527 pretreatment (Figures $5 d-f$ ). Notably, the Bcl-2/Bax ratio that is an 
a

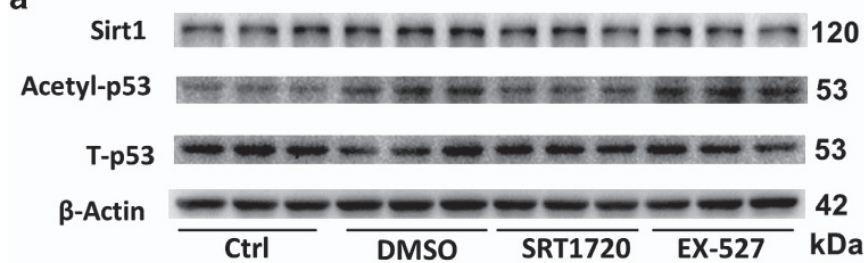

d

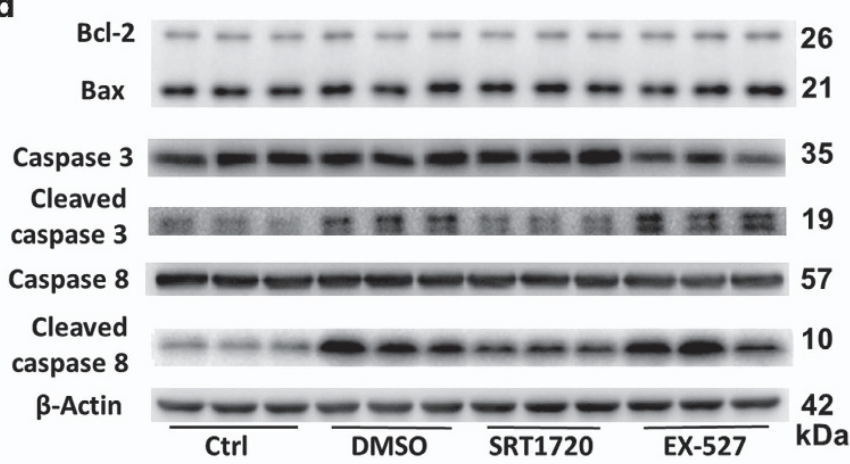

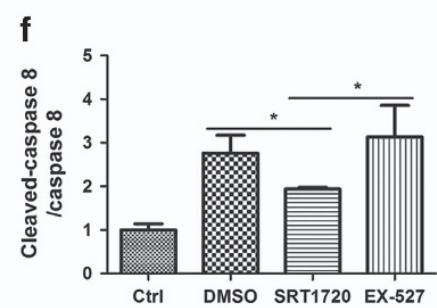

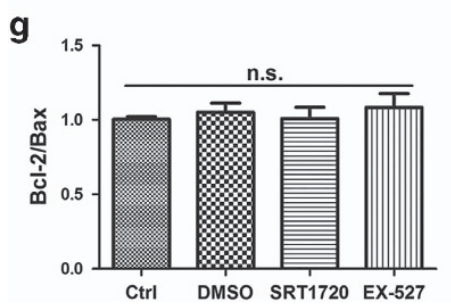

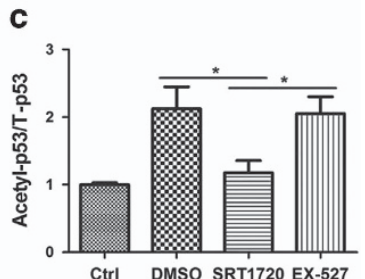

h

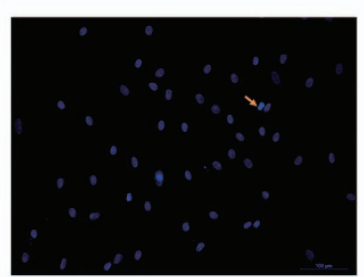

Ctrl

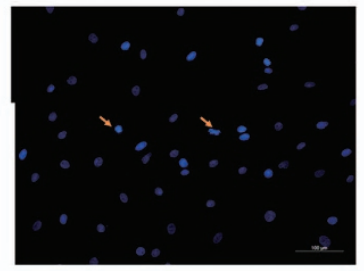

SRT1720

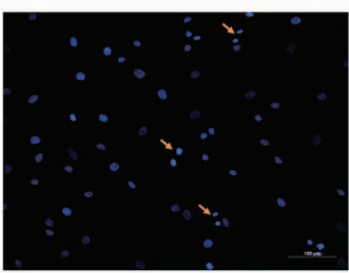

DMSO

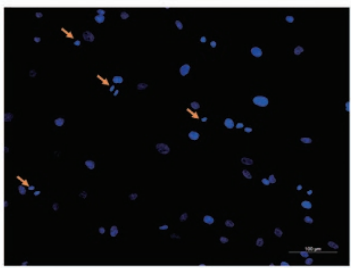

EX-527

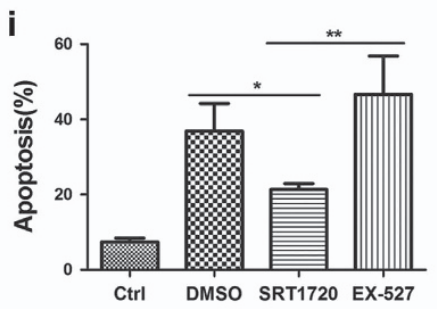

Figure 5 SRT1720 activates SIRT1 and protected aged hMSCs from apoptosis. SIRT1 expression (b) was detected by western blot (a), and SIRT1 activity was evaluated by the ratio of acety-p53/p53 (c) detected by western blot (a). Western blot (d) was carried out to quantify the apoptosis-associated protein cleaved caspase-3 (e), cleaved caspase-8 (f) involved in extrinsic apoptosis pathway and Bcl-2/Bax (g) involved in intrinsic apoptosis pathway. The apoptosis was also examined by Hoechst 33258 staining (h and i). Ctrl refers to normal culture medium. The other stress groups are treated with serum deprivation plus $\mathrm{H}_{2} \mathrm{O}_{2}$ medium. The volume fraction of DMSO was the same in every groups. Data are expressed by mean \pm S.D. (three independent experiments, $N=3$ ). ${ }^{\star} P<0.05 ;{ }^{* *} P<0.01$

indicator of intrinsic apoptosis was not changed (Figures $5 d$ and g). These results suggest that SRT1720 promotes survival of aged hMSCs by inhibiting the extrinsic apoptosis and is dependent on SIRT1 activation.

FAIM is necessary for the protective effects of SRT1720 on aged hMSCs. To further elucidate the mechanisms by which SRT1720 exerts its anti-apoptotic effect, we performed the transcriptome analysis of aged hMSCs pretreated with DMSO or SRT1720 for $24 \mathrm{~h}$ and then subjected to $0.5 \mathrm{mM}$ $\mathrm{H}_{2} \mathrm{O}_{2}$ for $3 \mathrm{~h}$. We identified 699 upregulated genes (>2-fold) and 645 downregulated genes $(<0.5$ fold) in the DMSO$\mathrm{H}_{2} \mathrm{O}_{2}$ culture group (HD group) compared to DMSO with normal culture group (ND group) (Supplementary figure 3c). Compared to the HD group, there were 471 upregulated and
494 downregulated genes in the group of aged hMSCs with SRT1720 pretreatment plus $\mathrm{H}_{2} \mathrm{O}_{2}$ stress (HS group) (Supplementary figure $3 \mathrm{c}$ ). Genes with dramatic changes in both HD versus ND groups and in HS versus HD groups are listed in the heat map (Supplementary Figure 3d). Among these, seven genes were associated with cell survival and apoptosis. We then validated these findings by quantitative real time PCR. Pro-apoptosis genes TNFRSF19 and TNFRSF18 were upregulated in serum deprivation with $\mathrm{H}_{2} \mathrm{O}_{2}$ culture group compared to normal culture and downregulated after SRT1720 pretreatment compared to vehicle control. Conversely, the anti-apoptosis gene FAIM and prosurvival or cell cycle genes TRAIP, SPDYA were downregulated in serum deprivation with $\mathrm{H}_{2} \mathrm{O}_{2}$ culture group compared to normal culture, and upregulated after SRT1720 
a

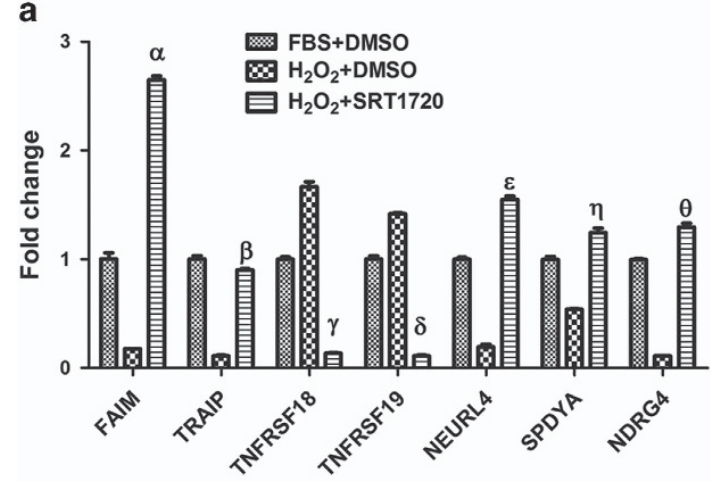

d

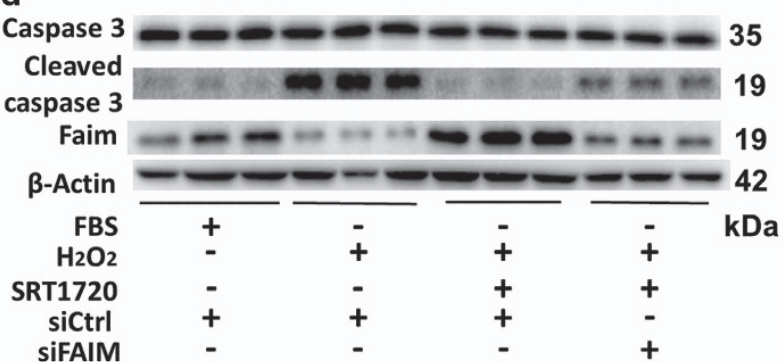

g

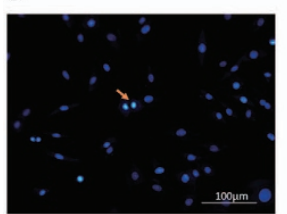

Ctrl

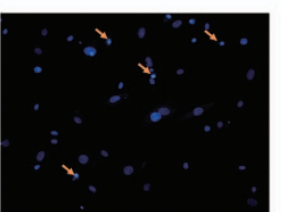

DMSO

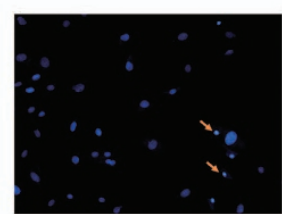

SRT1720 e
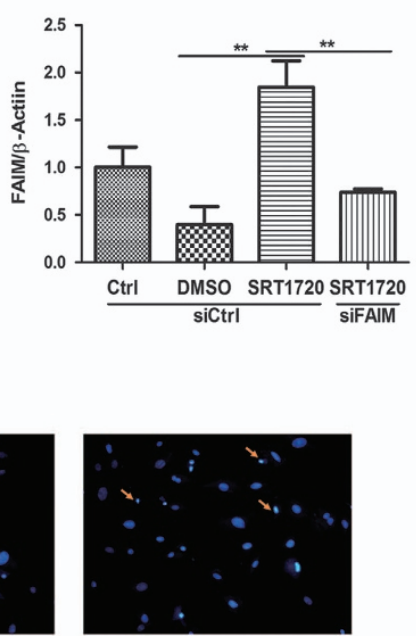

SRT1720

SiFAIM

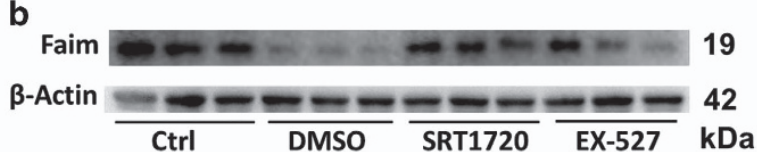

C

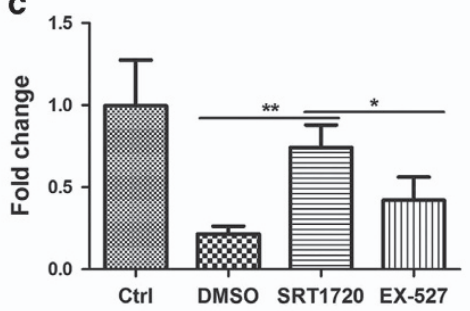

Figure 6 SRT1720 pretreatment protects aged hMSCs from apoptosis through upregulating FAIM. The expression of genes associated with cell survival and proliferation were confirmed by real time PCR (a). The expression of FAIM after SRT1720 pretreatment was tested by western blot (b and $\mathbf{c})$. The reversal of the changed induced by SRT1720 was also evaluated when FAIM was silenced with western blot $(\mathbf{d}-\mathbf{f})$. Representative images of Hoechst 33258 staining $(\mathbf{g})$ and quantitative analysis $(\mathbf{h})$ are shown for apoptosis examination. Ctrl refers to normal culture medium. The other stress groups are treated with serum deprivation plus $\mathrm{H}_{2} \mathrm{O}_{2}$ medium. The concentration of DMSO (solvent of SRT1720 and EX-527) was the same in every groups. Greek letters refer to the fold change in $\mathrm{H}_{2} \mathrm{O}_{2}+\mathrm{SRT} 1720$ group versus $\mathrm{H}_{2} \mathrm{O}_{2}+\mathrm{DMSO}$ group, $\alpha=14.89 \pm 0.24$; $\beta=8.07 \pm 0.19 ; \gamma=12.34 \pm 0.31 ; \delta=12.64 \pm 0.21 ; \varepsilon=8.05 \pm 0.25 ; \eta=2.31 \pm 0.22 ; \theta=11.64 \pm 0.27$. Data are expressed by mean \pm S.D. (three independent experiments, $N=3) .{ }^{*} P<0.05 ;{ }^{* *} P<0.01$

pretreatment compared to vehicle control (Figure 6a). The anti-apoptosis gene FAIM demonstrated the most dramatic change in expression following SRT1720 pretreatment $(14.9 \pm 0.2$ folds versus DMSO pretreated group), and may account at least in part for the anti-apoptosis effects produced by SRT1720. Upregulation of FAIM was also observed at the protein level in aged hMSCs pretreated with SRT1720, while reduced protein expression of FAIM was observed in aged hMSCs pretreated with EX-527, as expected (Figures $6 \mathrm{~b}$ and $\mathrm{c}$ ).

To investigate whether FAIM mediated the anti-apoptosis effect of SRT1720 in aged hMSCs induced by $\mathrm{H}_{2} \mathrm{O}_{2}$, a small interfering RNA targeted FAIM (siFAIM) was used. Compared to SRT1720 pretreatment group, the ratio of apoptosis cells in SRT1720 combined with siFAIM pretreatment group were significantly increased (Figures $6 \mathrm{~g}$ and $\mathrm{h}$ ) and activation of caspase- 3 was also upregulated (Figures $6 d-f$ ). These results reveal that downregulation of FAIM mitigated the antiapoptosis actions of SRT1720. Taken together, these results suggest that FAIM is necessary to mediate the protection of SRT1720 in aged hMSCs under oxidative stress.

\section{Discussion}

Here we have shown that aged hMSCs have increased sensitivity to oxidative stress and this correlates with significantly decreased activity and expression of SIRT1. On the basis of these results and our previous reports, ${ }^{15,16}$ we speculated that SIRT1 has an important role in the reduced stress resistance of aged hMSCs and the subsequent low survival of aged MSCs following cell transplantation in vivo. In support of this, we found that optimal treatment of aged hMSCs with the SIRT1 agonist SRT1720 enhanced cell survival in cultured cells under conditions of hypoxia and 
oxidative stress that mimic in part the conditions of ischemia and reperfusion that are present during $\mathrm{Ml}$ in vivo. Enhanced survival in vitro was paralleled by enhanced cell therapy of aged hMSCs by SRT1720 pretreatment in a rat MI model of permament LAD ligation. H-MSCs pretreated with SRT1720 under optimal conditions conferred enhanced early survival and engraftment with lower scar and fibrosis and marked improvement of cardiac contractility at later time. We provide evidence that the enhanced functions conferred by SRT1720 involve enhanced activity but not expression of SIRT1 and improved protection against apoptosis perhaps mediated by enhanced expression of the FAIM by aged hMSCs induced by SRT1720 through SIRT1 in the aged cells. The results suggest a pharmacological approach to improve the potential of aged hMSCs for the treatment of cardiovascular disease including heart disease and stroke.

MSCs therapy for cardiovascular disease is through to be limited to a significant degree by the poor survival and engraftment of cells after transplantation, effects that are exacerbated by age. ${ }^{5,6}$ In recent years, a variety of approached have been developed to improve such survival and engraftment of transplanted MSCs. Retroviral-mediated overexpression of the pro-survival gene Akt1 was first shown to enhance the survival of MSCs in an ischemic setting. ${ }^{23}$ Hypoxic pre-conditioning has also been used to improve the survival of multiple cell types after transplantation including MSCs. ${ }^{24,25}$ Similarly, a variety of small molecules that enhance survival by blocking apoptosis have been shown to enhance MSCs engraftment and improve therapeutic efficacy. ${ }^{26,27}$ To date and to our knowledge, no strategy has been described to specifically improve the survival of aged hMSCs in the setting of ischemic heart disease. Therefore, work described here is novel and significant.

Of all the synthesized SIRT1 activators, SRT1720 appears to be the most effective and shows promise in clinical application19. However, the actions of SRT1720 are cellspecific. SRT1720 has been shown to decrease the viability of multiple myeloma cells through caspase-3 mediated apoptosis $^{28}$ and pancreatic cancer cells through a SIRT1lysosomal-cell death pathway. ${ }^{29}$ SRT1720 appears to have a mainly protective effect in non-tumor cells. For example, SRT1720 ameliorated the senescence of airway epithelial cells, ${ }^{30}$ attenuated the endoplasmic reticulum stress and superoxide production of vascular endothelial cells, ${ }^{31,32}$ and induced mitochondrial biogenesis in renal proximal tubule cells after oxidant injury. ${ }^{33}$ The latter results are consistent with our findings that SRT1720 protects aged hMSCs during subjection to ischemic/oxidative stress perhaps by suppressing the extrinsic apoptosis pathway and associated caspase8 and its downstream caspase- 3 .

Our studies identify FAIM, an anti-apoptosis protein as the possible target for SIRT1 and mediator of cytoprotection by SRT1720. FAIM was first discovered in Fas-resistant B lymphocytes and is broadly expressed in multiple tissues. ${ }^{34}$ The sequence of FAIM gene is evolutionarily conserved from C. elegans to mammalian species, which supports a central role for the FAIM gene as a key apoptotic regulatory molecule. $^{34}$ FAIM has an important role in altering the expression of c-FLIP and inhibiting fas-mediated apoptosis by binding to Fas to block its role in activating caspase-8 (in our studies, we found that the expression of apoptosis-associated cleaved caspase-3 and cleaved caspase-8 were decreased in SRT1720 pretreated aged hMSCs coincident with an upregulation of FAIM (Figures 5 and 6). ${ }^{35}$ A central role for FAIM was confirmed by our demonstration that siRNA-mediated downregulation of FAIM mitigated the anti-apoptosis effect of SRT1720 (Figure 6). These results confirm that FAIM is necessary for the protective effects of SRT1720.

Conclusion and perspectives. We describe an effective pharmacological approach to pretreat aged hMSCs with SRT1720 that improves cell survival under oxidative stress and provides enhanced cell therapy. SRT1720 pretreated aged hMSCs demonstrated significantly improved survival and engraftment in ischemic hearts that lead to markedly preserved myocardial functions post MI. Our results suggest this modality of pretreatment has promising implications for clinical studies. It was recently demonstrated that SIRT1 and systemic SRT1720 conferred cardioprotection in a ischemiareperfusion model. ${ }^{36-38}$ Therefore, it may even be feasible to combine pretreatment of MSCs with systemic administration of SRT1720 to provide an optimal therapy for MI. Our in vivo results are the first to describe safety and efficacy of SRT1720 pretreated aged MSCs in an MI model. The results support implementation of further mechanistic, pharmacological and toxicological studies in a large animal model prior to possible clinical application of this technology.

\section{Materials and Methods}

Animals and human bone marrow. Sprague-Dawley (SD) rats were purchased from the Zhejiang Academy of Medical sciences (Hangzhou, China). All experimental protocols were approved by the animal ethics committee of the second affiliated hospital of Zhejiang University in accordance with the Guide for the Care and Use of Laboratory Animals (NIH Publication No. 85-23, revised 1996). Human bone marrows were harvested from healthy donors or patients undergoing total hip replacement surgery ( 3 young healthy donors and 3 elder patients, as described in Supplementary Table 1). Informed consent was obtained from each donor and the protocol of processing human cells was approved by the ethics committee of the second affiliated hospital of Zhejiang University.

Isolation and culture of MSCs. Bone marrows obtained from the proximal femur were washed with phosphate buffered saline (PBS), and mononuclear cells were separated using the bone marrow and cord blood nuclear cells isolation reagents (Wealthlin Science and Technologies, Toronto, Ontario, Canada) according to the user's guide. Then the collected MSCs enriched mononuclear cells were resuspended in low glucose Dulbecco's modified eagle medium (DMEM) (GIBCO, Life Technologies, Waltham, MA, USA) with $15 \%$ fetal bovine serum (FBS) (GIBCO, Life Technologies, USA). The cultures were plated on $10 \mathrm{~cm}$ dish and maintained in a humidified atmosphere containing $5 \% \mathrm{CO}_{2}$ at $37^{\circ} \mathrm{C}$. After $24 \mathrm{~h}$, non-adherent cells were removed and the remaining cells were cultured continuously. Cells were passaged at $70-80 \%$ confluence using $0.25 \%$ trypsin plus $0.2 \%$ EDTA and the $2-6$ passage cells were used for experiments.

Characterization of MSCs. H-MSCs at 6 passages were characterized for surface markers by a FACS Canto II Flow Cytometer (BD Bioscience, San Jose, CA, USA). Briefly, $1 \times 10^{6}$ cells were collected and suspended in $100 \mu \mathrm{l}$ PBS with $1 \%$ FBS, and then stained with the surface molecular specific antibodies (mesenchymal surface markers: PE-CD29, PE-CD44, APC-CD90, endothelial cell surface marker: FITC-CD34, hematopoietic surface marker: FITC-CD45 and isotype-matched control) for $1 \mathrm{~h}$. After twice wash with PBS, the expression of cell surface markers was quantified with FACSDiva software (BD Bioscience).

The differentiation of hMSCs into osteocytes, chondrocytes and adipocytes was conducted by the methods used in our previous study. ${ }^{39}$ Briefly, hMSCs were seeded in 6-well plate at density of $2 \times 10^{5}$ per well, cultured until confluent. For the 
differentiation into adipocytes, hMSCs were treated with the adipogenesis induction medium for 23 days. The formation of fat vacuoles was visualized by oil red 0 staining. For osteogenesis differentiation, hMSCs were treated with osteogenesis induction medium for 3 weeks. Osteogenesis of hMSCs was evaluated with alizarin red S staining. Chondrogenesis differentiation of hMSCs was induced with chondrogenic induction medium for 3 weeks, and evaluated with toluidine blue staining. Images were captured with a light microscope.

Establishment of cell stress models and cell survival assay. According to the commonly used models, three stress situations mimicking in vivo ischemic condition were developed in vitro. Aged hMSCs cultured in 96-well plate were exposed to normal culture or serum deprivation culture, serum deprivation culture under $0.1 \% \mathrm{O}_{2}$ and serum deprivation culture with $\mathrm{H}_{2} \mathrm{O}_{2}$ for different time points. To maximize the effects of SRT1720, the appropriate conditions for stress were obtained when $50 \%$ cells survived under these stresses compared to normal culture. Cell survival was detected by cell counting kit 8 (CCK-8). Briefly, the medium was removed after stress and $100 \mu$ l DMEM with $10 \mu$ l CCK-8 (Dojindo, Minato-ku, Tokyo, Japan) solution were added to each well. After incubation for $2.5 \mathrm{~h}$ at $37^{\circ} \mathrm{C}$, the absorbance at $450 \mathrm{~nm}$ was measured by a microplate reader (Bio-Rad, Berkeley, CA, USA).

Senescence-associated $\boldsymbol{\beta}$-galactosidase staining. H-MSCs of the same passage (from younger and elder patients) were trypsinized and seeded into 6 -well plate at $2 \times 10^{5}$ cells per well. The next day, culture mediums were removed and cells were washed with PBS for three times and fixed with $4 \%$ formaldehyde for $15 \mathrm{~min}$. After washing with PBS for three times, $\beta$-galactosidase staining was conducted with cell senescence-associated $\beta$-galactosidase staining kit (Byotime Biotechnology, Shanghai, China) per manufacturer's instructions. Cells were incubated at $37{ }^{\circ} \mathrm{C}$ overnight and washed with PBS for three times. Then imaging was performed with the inverted microscope (Leica, Wetzlar, Germany).

SIRT1 activity assay. The nuclear protein was extracted from cells using Nuclear and Cytoplasmic Protein Extraction Kit (Beyotime Biotechnology, Shanghai, China) according to the manufacturer's protocols. The protein concentrations were determined by BCA method (Thermo Fisher Scientific, Waltham, MA, USA), and equal protein amounts from different samples were used to test the activity of SIRT1 according to the instruction of SIRT1 Fluorometric Drug Discovery Kit (Enzo Life Sciences, Farmingdale, NY, USA). Briefly, the nuclear protein solutions were incubated with standard acetylated substrate p53 protein $(100 \mu \mathrm{M})$ and co-substrate $\mathrm{NAD}^{+}(170 \mu \mathrm{M})$ for $45 \mathrm{~min}$ at $37^{\circ} \mathrm{C}$, and then developer II was added to the system for another $45 \mathrm{~min}$ to terminate the reactions. The fluorescence intensity at $460 \mathrm{~nm}$ excited by $360 \mathrm{~nm}$ light was measured using a fluorescence microplate reader (Molecular Devices, Sunnyvale, CA, USA).

Pharmacological treatment of hMSCs. The SIRT1 specific activator SRT1720 and inhibitor EX-527 were all purchased from Selleck Chemicals (Houston, TX, USA), and dissolved into an organic solvent, dimethylsulfoxide (DMSO) before the treatment of hMSCs. To avoid the solvent disturbance of DMSO, concentrations of DMSO are less than $0.1 \%$ and the same in different treatment groups. Cells were exposed to fresh complete medium supplemented with different concentration of SRT1720 for different time points, followed by treatment with different cell stress models, to evaluate the optimal SRT1720 concentration and optimal timepoint by using CCK-8 test. For in vivo experiment, aged hMSCs were cultured in $10 \mathrm{~cm}$ dishes before treatment. When cultured to $80 \%$ confluence, cells were pretreated with (SRT1720 group) or without SRT1720 (DMSO group), while the concentration of solvent DMSO is $<0.1 \%$ and the same in both groups. After pretreated for $24 \mathrm{~h}$, cells were collected in DMEM for implantation into rat hearts.

Hoechst staining. For apoptosis detection, hMSCs were trypsinized and seeded into 24-well plate at 20000 cells per well. After treatments, culture mediums were removed and cells were rinsed twice with PBS. Cells were fixed with $4 \%$ formaldehyde for $15 \mathrm{~min}$ followed by washing with PBS for three times. Overall, $100 \mu \mathrm{l}$ of $10 \mu \mathrm{g} / \mathrm{ml}$ Hoechst 33258 (Life Technology, USA) was added and incubated at room temperature for $15 \mathrm{~min}$. After three washes with PBS, the images were photographed with a florescence microscope (Leica, Wetzlar, Germany).

RNA extraction and transcriptional profile analysis. Total RNA was isolated from hMSCs immediately after treatments using Trizol regent (Invitrogen, Carlsbad, CA, USA) according to the instructions. The concentrations and qualities of RNA were examined with NanoDrop 2000c spectrophotometer (Thermo Fisher Scientific, Waltham, MA, USA). Gene expression microarray was conducted with the Illumina Whole-Genome Gene Expression Direct Hybridization Assay system (Illumina, SanDiego, CA, USA). In brief, $500 \mathrm{ng}$ of total RNA were reverse transcribed and synthesized to double-stranded complementary DNA (cDNA). After purification, cDNA were used to amplify and label multiple copies of biotinylated cRNA by in vitro transcription. After quantification, the labeled cRNA were dispensed to BeadChips and incubated for $14-20 \mathrm{~h}$ at $58^{\circ} \mathrm{C}$. After washing, the BeadChip was bind by Cy3-SA and the signal was detected by the iScan System and BeadArray Reader (Illumina, SanDiego, CA, USA). The gene expression data from scanned microarray was analyzed using Illumina's Genome Studio Gene Expression Module. The raw data were normalized using the quantile method to minimize the effects of variation arising from non-biological factors. Differential expression algorithms were used to compare a group of samples (referred to as the condition group) to a reference group, and the comparison was done using the error model of detection $P$-value. Differential expression was defined by fold change between groups which was more than 2 or $<0.5$. Clustering analysis was also conducted to examine the similarity of differential expression genes (Supplementary Figures $2 \mathrm{~A}$ and $\mathrm{B}$ ).

Small interfering RNA transfection. Small interfering RNA (siRNA) was applied to silencing the expression of FAIM, and lipofectamine 2000 regent (Life Technology, Waltham, MA, USA) was used to transfect the FAIM specific siRNA (5'-GGGUGAGUUUGUAGAUGAUTT-3') and negative control siRNA (5'-UU CUCCGAACGUGUCACGUTT-3') into hMSCc according to the manufacturer's instructions. The optimized concentration of FAIM specific siRNA for transfection was determined by real time PCR and western blot. In brief, 1 day prior to siRNA transfection, hMSCs were plated into 6-well plates in normal medium to attain 50$60 \%$ confluence when transfecting the next day. Then, 50 pmol of siRNA with $250 \mu \mathrm{l}$ Opti-MEM (Life Technologies) and $2.5 \mu$ lof lipofectamine 2000 (Life Technologies) with $250 \mu \mathrm{l}$ Opti-MEM were mixed for $20 \mathrm{~min}$ at room temperature and added to each well. After incubation for $5 \mathrm{~h}$, the complexes were removed and fresh medium with $15 \%$ FBS were added. Overall, $24 \mathrm{~h}$ after transfection, total RNA were extracted from hMSCs and tested by real time PCR. Total protein was isolated and detected by western blot $48 \mathrm{~h}$ after transfection.

Quantitative real time RT-PCR. Prior to PCR, $1 \mu \mathrm{g}$ of RNA was reverse transcribed to cDNA using the PrimeScript RT reagent Kit (Perfect Real Time, Takara, Japan) per manufacturer's instructions. cDNA was diluted by 5 -folds and used for quantitative real time PCR using the SYBR Premix Ex Taq $(2 x)$ reaction mix (Takara, Japan) on the 7500 Fast real time PCR system (Applied Biosystems, Carlsbad, CA, USA). The reaction conditions included: $95^{\circ} \mathrm{C}$ for $10 \mathrm{~min}$ and then 40 cycles of $95^{\circ} \mathrm{C}$ for $15 \mathrm{~s}, 65^{\circ} \mathrm{C}$ for $30 \mathrm{~s}$ and $72^{\circ} \mathrm{C}$ for $10 \mathrm{~s}$. Primers were designed with the Primer 3 Input (version 4.0.0) online software (http://primer3.ut.ee/) and the sequences were listed in Supplementary Table 2. The expression of target genes was determined by the comparative $\Delta \Delta \mathrm{Ct}$ method and $\beta$-ACTIN was used as the control gene.

Western blotting analysis. Cells were homogenized in ice-cold lysis buffer supplemented with proteinase inhibitor. After $30 \mathrm{~min}$ ' incubation, cell lysates were centrifuged at $12000 \mathrm{~g}$ for $30 \mathrm{~min}$ at $4^{\circ} \mathrm{C}$ to remove debris. Protein concentrations were measured by BCA method (Thermo Fisher Scientific), separated by sodium dodecyl sulfate polyacrylamide gel electrophoresis (SDS-PAGE) with a current of 30 $\mathrm{mA}$ and transferred onto polyvinylidene difluoride (PVDF) membranes (Merck Millipore, Darmstadt, Germany) electrophoretically with a current of $300 \mathrm{~mA}$. After blocking with $5 \%$ bovine serum albumin (BSA) for $1 \mathrm{~h}$, the membranes were incubated with primary antibodies at a concentration according to the product instructions overnight at $4{ }^{\circ} \mathrm{C}$. The membranes were washed three times with PBSTween (PBST) and incubated with corresponding HRP-conjugated secondary antibodies (1:5000) for $1 \mathrm{~h}$ at room temperature. After being washed three times with PBST, the protein bands were detected using the Gel Doc EZ Imaging System (Bio-Rad, Berkeley, CA, USA) with an ECL kit (Merck Millipore, Germany) and analyzed using Image Lab software (Bio-Rad).

Mouse monoclonal anti-SIRT1 antibody, Mouse monoclonal anti-cleaved-caspase8 and rabbit monoclonal anti-acetyl-p53 (K382) antibody were obtained from Abcam (Cambridge, UK). Rabbit monoclonal anti-Bcl-2 antibody, rabbit monoclonal anti-Bax antibody, rabbit monoclonal anti-cleaved-caspase-3 antibody and rabbit monoclonal anti-FAIM antibody were obtained from Cell Signaling Technology (Beverly, MA, USA). Mouse monoclonal $\beta$-actin antibody was obtained from BD Biosciences 
(San Jose, CA, USA), horseradish peroxidase (HRP)-conjugated anti-mouse and anti-rabbit secondary antibodies were obtained from Cell Signaling Technology.

MI model and hMSCs transplantation. Rats MI model were established by ligation of the left anterior descending $(L A D)$ coronary artery of female $S D$ rats (8 weeks old, 220-250 g) as previously described. ${ }^{40}$ All 60 female SD rats were randomly divided into four groups: the sham group (sham) received the same thoracotomy surgery without ligaturing the LAD coronary or any other intervention $(n=15)$; the DMEM group (DMEM) received coronary ligation and DMEM injection $(n=15)$; the DMSO pretreated aged MSCs group (DMSO-OMSCs) received coronary ligation and injection of male aged hMSCs pretreated with DMSO $(n=15)$; the SRT1720 pretreated aged MSCs group (SRT1720-OMSCs) received coronary ligation and injection of male aged hMSCs pretreated with SRT1720 $(n=15)$. Rats $\mathrm{Ml}$ model were established by ligation of the LAD descending coronary artery of female SD rats (8 weeks old, 220-250 g). In brief, the hearts of rats were exposed by limited thoracotomy with tracheal intubation under general anesthesia with pentobarbital $(50 \mathrm{mg} / \mathrm{kg})$, and the LAD coronary artery was ligatured with a 6-0 silk suture. After $30 \mathrm{~min}$, the ischemic border zone of hearts were intramyocardially injected with $150 \mu$ l DMEM or 10 million aged male hMSCs using a 30-gauge needle at five sites. After the surgery, the chest was closed and a warming pad was arranged for the recovery of rats.

Measurement of SRY gene by quantitative real time PCR. Human sex determination SRY gene copy number in female rat heart tissues following hMSCs transplantation was measured by real time PCR. In brief, the whole heart tissues from different groups were frozen and pulverized with liquid nitrogen. Genomic DNA was extracted using MiniBEST Universal Genomic DNA Extraction Kit Ver.5.0 (Takara, Japan) according to the manufacturer's instructions. Real time PCR was performed with SYBR green reaction mix using 7500 fast real time PCR system (Applied Biosystems, Carlsbad, CA, USA) according to manufacturer's instructions. Primers used include: human SRY gene forward primer $5^{\prime}$ GGTAAGTGGCCTAGCTGGTG $3{ }^{\prime}$, reverse primer 5'GATCCCGCTTCGGTACT CTG $3^{\prime}$; rat 36B4 gene: forward primer 5'-CTCACTCCATCATCAATGGATAC AA-3', reverse primer $5^{\prime}$-CAGCCAGTGGGAAGGTGTAGTCA-3'. The reaction conditions were $95^{\circ} \mathrm{C}$ for $30 \mathrm{~s}$ followed by 40 cycles of $95^{\circ} \mathrm{C}$ for $5 \mathrm{~s}, 56^{\circ} \mathrm{C}$ for $31 \mathrm{~s}$ and $95^{\circ} \mathrm{C}$ for $15 \mathrm{~s}$. Engrafted hMSCs numbers were evaluated using the comparative $\Delta \Delta$ Ct comparative method.

Echocardiographic examination. Cardiac function of rats at the 28th day following cell therapy were evaluated by transthoracic two dimensional echocardiography (Vevo 2100; VisualSonics, Toronto, Ontario, Canada) under general anesthesia with $2 \%$ isoflurane in $\mathrm{O}_{2}$ gas. The measurement of LVEDD, LVESD were conducted in at least three cycles. LVESV and LVEDV were calculated according to the M-mode formula of Teichholtz: $7 \times$ LVEDD $^{3} /(2.4+L V E D D)$ and

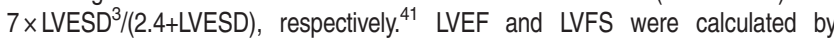
((LVEDD - LVESD)/LVEDD $) \times 100$ and ((LVEDV - LVESV)/LVEDV) $\times 100$, respectively. The echocardiographic analysis was performed in a double-blinded manner. The animal surgery, echocardiographic examination, and data analysis were conducted by three different researchers, respectively.

Masson's trichrome staining. Scar size of the rat hearts were evaluated by Masson's Trichrome staining as previously described. ${ }^{42}$ In brief, hearts were collected from rats of different groups and made into frozen sections of $7 \mu \mathrm{m}$. Then Masson's Trichrome staining was performed using Masson Trichrome Kit (Sigma Aldrich, St. Louis, MO, USA) according to the manufacturer's instructions. The percentage of fibrosis in the whole left ventricular was quantified with ImagePro-Plus software (Media Cybernetics, Rockville, MD, USA).

Immunofluorescence staining. Frozen sections from rat hearts were fixed with $4 \%$ formaldehyde for 15 min and permeabilized with $0.25 \%$ Triton X100 for $20 \mathrm{~min}$. The slices were washed with $0.05 \%$ PBST for three times and blocked with $5 \%$ BSA in PBST for $1 \mathrm{~h}$. The sections were incubated with specific primary antibody $(1: 100)$ overnight at $4^{\circ} \mathrm{C}$. After three washes in PBST, the sections were incubated with the corresponding secondary antibody for 1 hour at room temperature. After three washes, the nucleus was stained with 4',6-diamidino-2phenylindole (DAPI). Fluorescent imaging was performed using a fluorescent microscope (Leica, Wetzlar, Germany). The primary and secondary antibodies used in this experiment were obtained from Abcam (Cambridge, UK).
Statistical analysis. All data are shown as mean \pm S.D. and analysis was implemented with Graphpad Prism 5 software (GraphPad Software, La Jolla, CA, USA). A normal distribution was confirmed by the Shapiro-Wilk test for each set of the obtained data from each studied group. Student's $t$-test was used to compare between two groups, and one-way analysis of variance (ANOVA) was performed to analyze the difference among more than two groups followed by multiple comparisons using the Student-Newman-Keuls test. Differences were considered significant when $P<0.05$.

\section{Conflict of Interest}

The authors declare no conflicts of interest.

Acknowledgements. We thank professor Keith A. Webster for his English modification of our manuscript. This work was supported by the National Basic Research Program of China (973 Program, Nos. 2014CB965100, 2014CB965102, 2014CB965103 for JW), National High-tech R\&D 863 Program (No. 2013AA020101 for XH, No. 2015AA020922 for XL), grants from National Natural Science Foundation of China (Nos. 81320108003,31371498 for JW, No. 81570233 for XL, No. 81370247 for $\mathrm{XYH}$, No. for LZ), Zhejiang Provincial Natural Science Foundation (No. LY16H280003 for LZ), and Science and Technology Department of Zhejiang province public welfare projects (No. 2014C33190 for RW).

1. Tse HF, Kwong YL, Chan JK, Lo G, Ho CL, Lau CP. Angiogenesis in ischaemic myocardium by intramyocardial autologous bone marrow mononuclear cell implantation. Lancet 2003; 361: $47-49$.

2. Wollert KC, Meyer GP, Lotz J, Ringes-Lichtenberg S, Lippolt P, Breidenbach C et al. Intracoronary autologous bone-marrow cell transfer after myocardial infarction: the BOOST randomised controlled clinical trial. Lancet 2004; 364: 141-148.

3. Williams AR, Trachtenberg B, Velazquez DL, McNiece I, Altman P, Rouy D et al. Intramyocardial stem cell injection in patients with ischemic cardiomyopathy: functional recovery and reverse remodeling. Circ Res 2011; 108: 792-796.

4. Choudry F, Hamshere S, Saunders N, Veerapen J, Bavnbek K, Knight C et al. A randomized double-blind control study of early intra-coronary autologous bone marrow cell infusion in acute myocardial infarction: the REGENERATE-AMI clinical trialdagger. Eur Heart J 2016; 14: 256-263.

5. Liang H, Hou H, Yi W, Yang G, Gu C, Lau WB et al. Increased expression of pigment epithelium-derived factor in aged mesenchymal stem cells impairs their therapeutic efficacy for attenuating myocardial infarction injury. Eur Heart J 2013; 34: 1681-1690.

6. Fan M, Chen W, Liu W, Du GQ, Jiang SL, Tian WC et al. The effect of age on the efficacy of human mesenchymal stem cell transplantation after a myocardial infarction. Rejuvenation Res 2010; 13: 429-438.

7. Zhou S, Greenberger JS, Epperly MW, Goff JP, Adler C, Leboff MS et al. Age-related intrinsic changes in human bone-marrow-derived mesenchymal stem cells and their differentiation to osteoblasts. Aging Cell 2008; 7: 335-343.

8. Stolzing A, Jones E, McGonagle D, Scutt A. Age-related changes in human bone marrowderived mesenchymal stem cells: consequences for cell therapies. Mech Ageing Dev 2008; 129: 163-173.

9. Zaim M, Karaman S, Cetin G, Isik S. Donor age and long-term culture affect differentiation and proliferation of human bone marrow mesenchymal stem cells. Ann Hematol 2012; 91: $1175-1186$.

10. Boily G, Seifert EL, Bevilacqua L, He XH, Sabourin G, Estey $C$ et al. SirT1 regulates energy metabolism and response to caloric restriction in mice. PLOS ONE 2008; 3: e1759.

11. Herskovits AZ, Guarente L. SIRT1 in neurodevelopment and brain senescence. Neuron 2014; 81: 471-483

12. Yeung F, Hoberg JE, Ramsey CS, Keller MD, Jones DR, Frye RA et al. Modulation of NF-kappaB-dependent transcription and cell survival by the SIRT1 deacetylase. EMBO J 2004; 23: 2369-2380.

13. Luo J, Nikolaev AY, Imai S, Chen D, Su F, Shiloh A et al. Negative control of p53 by Sir2alpha promotes cell survival under stress. Cell 19, 2001; 107: 137-148.

14. Brunet A, Sweeney LB, Sturgill JF, Chua KF, Greer PL, Lin $Y$ et al. Stress-dependent regulation of FOXO transcription factors by the SIRT1 deacetylase. Science 26, 2004; 303: 2011-2015.

15. Chen H, Liu X, Zhu W, Chen H, Hu X, Jiang Z et al. SIRT1 ameliorates age-related senescence of mesenchymal stem cells via modulating telomere shelterin. Front Aging Neurosci 2014; 6: 103

16. Liu X, Chen H, Zhu W, Chen H, Hu X, Jiang Z et al. Transplantation of SIRT1-engineered aged mesenchymal stem cells improves cardiac function in a rat myocardial infarction model. J Heart Lung Transplant 2014; 33: 1083-1092.

17. Howitz KT, Bitterman KJ, Cohen HY, Lamming DW, Lavu S, Wood JG et al. Small molecule activators of sirtuins extend Saccharomyces cerevisiae lifespan. Nature 2003; 425: 191-196.

18. Hubbard BP, Sinclair DA. Small molecule SIRT1 activators for the treatment of aging and age-related diseases. Trends Pharmacol Sci 2014; 35: 146-154. 
19. Milne JC, Lambert PD, Schenk S, Carney DP, Smith JJ, Gagne DJ et al. Small molecule activators of SIRT1 as therapeutics for the treatment of type 2 diabetes. Nature 29, 2007; 450: $712-716$.

20. Minor RK, Baur JA, Gomes AP, Ward TM, Csiszar A, Mercken EM et al. SRT1720 improves survival and healthspan of obese mice. Sci Rep 2011; 1: 70.

21. Mitchell SJ, Martin-Montalvo A, Mercken EM, Palacios HH, Ward TM, Abulwerdi G et al. The SIRT1 activator SRT1720 extends lifespan and improves health of mice fed a standard diet. Cell Rep 13, 2014; 6: 836-843.

22. Warboys CM, de Luca A, Amini N, Luong L, Duckles H, Hsiao S et al. Disturbed flow promotes endothelial senescence via a p53-dependent pathway. Arteriosclerosis, thrombosis, and vascular biology. Arterioscler Thromb Vasc Biol 2014; 34: 985-995.

23. Mangi AA, Noiseux N, Kong D, He H, Rezvani M, Ingwall JS et al. Mesenchymal stem cells modified with Akt prevent remodeling and restore performance of infarcted hearts. Nat Med 2003; 9: 1195-1201.

24. Hu X, Yu SP, Fraser JL, Lu Z, Ogle ME, Wang JA et al. Transplantation of hypoxiapreconditioned mesenchymal stem cells improves infarcted heart function via enhanced survival of implanted cells and angiogenesis. J Thorac Cardiovasc Surg 2008; 135: $799-808$.

25. Zhu W, Chen J, Cong X, Hu S, Chen X. Hypoxia and serum deprivation-induced apoptosis in mesenchymal stem cells. Stem Cells 2006; 24: 416-425.

26. Chang W, Song BW, Lim S, Song H, Shim CY, Cha MJ et al. Mesenchymal stem cells pretreated with delivered Hph-1-Hsp70 protein are protected from hypoxia-mediated cell death and rescue heart functions from myocardial injury. Stem Cells 2009; 27: 2283-2292.

27. Liu XB, Wang JA, Ji XY, Yu SP, Wei L. Preconditioning of bone marrow mesenchymal stem cells by prolyl hydroxylase inhibition enhances cell survival and angiogenesis in vitro and after transplantation into the ischemic heart of rats. Stem Cell Res Ther 2014; 5: 111.

28. Chauhan D, Bandi M, Singh AV, Ray A, Raje N, Richardson P et al. Preclinical evaluation of a novel SIRT1 modulator SRT1720 in multiple myeloma cells. Br J Haematol 2011; 155: 588-598.

29. Chini CC, Espindola-Netto JM, Mondal G, Guerrico AM, Nin V, Escande C et al. SIRT1 activating compounds (STACs) negatively regulate pancreatic cancer cell growth and viability through a SIRT1-lysosomal-dependent pathway. Clin Cancer Res 2016; 15: 2496-2507.

30. Yao H, Chung S, Hwang JW, Rajendrasozhan S, Sundar IK, Dean DA et al. SIRT1 protects against emphysema via FOXO3-mediated reduction of premature senescence in mice. J Clin Invest 2012; 122: 2032-2045.

31. Fiorentino TV, Procopio T, Mancuso E, Arcidiacono GP, Andreozzi F, Arturi F et al. SRT1720 counteracts glucosamine-induced endoplasmic reticulum stress and endothelial dysfunction. Cardiovasc Res 15, 2015; 107: 295-306.

32. Gano LB, Donato AJ, Pasha HM, Hearon CM Jr., Sindler AL, Seals DR. The SIRT1 activator SRT1720 reverses vascular endothelial dysfunction, excessive superoxide production, and inflammation with aging in mice. Am J Physiol Heart Circ Physiol 2014; 307: H1754-H1763.
33. Funk JA, Odejinmi S, Schnellmann RG. SRT1720 induces mitochondrial biogenesis and rescues mitochondrial function after oxidant injury in renal proximal tubule cells. J Pharmacol Exp Ther 2010; 333: 593-601.

34. Schneider TJ, Fischer GM, Donohoe TJ, Colarusso TP, Rothstein TL. A novel gene coding for a Fas apoptosis inhibitory molecule (FAIM) isolated from inducibly Fas-resistant B lymphocytes. J Exp Med 15, 1999; 189: 949-956.

35. Huo J, Xu S, Guo K, Zeng Q, Lam KP. Genetic deletion of faim reveals its role in modulating c-FLIP expression during CD95-mediated apoptosis of lymphocytes and hepatocytes. Cell Death Differ 2009; 16: 1062-1070.

36. Hsu CP, Zhai P, Yamamoto T, Maejima Y, Matsushima S, Hariharan N et al. Silent information regulator 1 protects the heart from ischemia/reperfusion. Circulation 2010; 122: 2170-2182.

37. Yamamoto T, Sadoshima J. Protection of the heart against ischemia/reperfusion by silent information regulator 1. Trends Cardiovasc Med 2011; 21: 27-32.

38. Tong C, Morrison A, Mattison S, Qian S, Bryniarski M, Rankin B et al. Impaired SIRT1 nucleocytoplasmic shuttling in the senescent heart during ischemic stress. FASEB J 2013; 27: 4332-4342.

39. Hu X, Xu Y, Zhong Z, Wu Y, Zhao J, Wang Y et al. A large-scale investigation of hypoxiapreconditioned allogeneic mesenchymal stem cells for myocardial repair in nonhuman primates: paracrine activity without remuscularization. Circ Res 2016; 118: 970-983.

40. Jiang Z, Hu X, Yu H, Xu Y, Wang L, Chen $\mathrm{H}$ et al. Human endometrial stem cells confer enhanced myocardial salvage and regeneration by paracrine mechanisms. J Cell Mol Med 2013; 17: 1247-1260.

41. Teichholtz LE, Kreulen T, Herman MV, Gorlin R. Problems in echocardiographic volume determinations: echocardiographic-angiographic correlations in the presence or absence of asynergy. Am J Cardiol 1976; 37: 7-11.

42. Hu X, Wu R, Jiang Z, Wang L, Chen $P$, Zhang $L$ et al. Leptin signaling is required for augmented therapeutic properties of mesenchymal stem cells conferred by hypoxia preconditioning. Stem Cells 2014; 32: 2702-2713.

(i) Cell Death and Disease is an open-access journal published by Nature Publishing Group. This work is licensed under a Creative Commons Attribution 4.0 International License. The images or other third party material in this article are included in the article's Creative Commons license, unless indicated otherwise in the credit line; if the material is not included under the Creative Commons license, users will need to obtain permission from the license holder to reproduce the material. To view a copy of this license, visit http://creativecommons.org/licenses/by/4.0/

(C) The Author(s) 2017

Supplementary Information accompanies this paper on Cell Death and Disease website (http://www.nature.com/cddis) 